\title{
Studies on the control of iron uptake by rabbit small intestine
}

\author{
BY T. M. COX AND M. W. O'DONNELL \\ Department of Medicine, Royal Postgraduate Medical School, \\ Ducane Road, London W12 OHS
}

(Received 29 June 1981-Accepted 12 November 1981)

1. Whole-body retention in vivo and uptake of ${ }^{59} \mathrm{Fe}$-labelled ascorbate and nitrilotriacetate chelates by intestinal slices in vitro were determined in groups of normal control rabbits and rabbits with experimentally-induced $\mathrm{Fe}$ deficiency.

2. Over-all absorption as measured by retention of doses of either chelate was greatly increased in conditions of Fe deficiency.

3. Intestinal $\mathrm{Fe}$ uptake in vitro was inhibited up to $77 \%$ in the presence of 2,4-dinitrophenol and sodium fluoride. Initial rates showed saturation within the concentration range $18-450 \mu \mathrm{mol} / 1$, suggesting that uptake was brought about by an active transport process.

4. When studied at chelate concentrations of $450 \mu \mathrm{mol} / 1$, significant regional differences in uptake rates were observed. Uptake in duodenal slices was increased when compared with slices from jejunum and ileum.

5. Fe uptake from ferric and ferrous chelates was greatly enhanced in Fe deficiency. This was chiefly due to increases in uptake by slices from the duodenum, but uptake into slices of distal intestine was also stimulated.

6. Kinetic analysis of $\mathrm{Fe}$ uptake by duodenal slices from animals rendered Fe deficient by diet or repeated bleeding indicated in both groups an increased apparent maximum velocity $\left(V_{\max }\right)$ for influx of $\mathrm{Fe}$ without significant changes in apparent affinity for Fe.

7. The experiments provide further insight into the nature and regional distribution of transport of Fe into the intestine and suggest, in the rabbit, that important control of Fe absorption may be exerted by an active process operating at this initial entry step.

Although it is generally accepted that the small intestine maintains iron homoeostasis by regulating absorption of dietary Fe to meet body needs (McCance \& Widdowson, 1937), the rate-limiting step in the transfer of Fe from lumen to the portal blood has yet to be defined. A priori, control of Fe absorption could be exerted at several loci within the luminal epithelium of the intestinal mucosa: at the brush border membrane; at intracellular sites of Fe transfer or at the level of efflux across the baso-lateral membrane.

Recent experiments employing biopsy specimens of human duodenal mucosa incubated in vitro with radio-labelled ferric chelates indicate that entry of $\mathrm{Fe}$ into the absorptive epithelium is a saturable process with features of active transport (Cox \& Peters, 1979). Moreover, in conditions of Fe deficiency, initial rates of Fe entry were reversibly stimulated, suggesting, in man, that the uptake step may be important in over-all control of Fe absorption (Cox \& Peters, 1980).

In this paper we report further observations in experimental preparations of animal intestine with special reference to modulations of mucosal Fe uptake occurring as adaptive responses to biological stress. The study provides additional evidence, in the rabbit, that control of over-all Fe absorption is exerted at the initial uptake step across the brush border, which may thus be a rate-limiting transport process.

\section{EXPERIMENTA L}

Animals

Healthy male New Zealand White Rabbits were obtained from Froxfield Laboratory Animal Breeders, Hants. Animals weighing approximately $2.5 \mathrm{~kg}$ were pair-matched for age and weight and caged individually. Weanling animals were used in nutritional studies at 8 weeks of age when they weighed approximately $1 \mathrm{~kg}$. 


\section{Induction of Fe deficiency}

Haemorrhagic anaemia was induced by twice-weekly venesection of blood $(10 \mathrm{ml} / \mathrm{kg}$ body-weight) for 4-6 weeks in one partner of eight matched pairs of animals. The animals were maintained on Labsure RAF diet, containing $230 \mathrm{mg} \mathrm{Fe} / \mathrm{kg}$.

Nutritional anaemia was induced in the group of weanling animals maintained for 3-4 weeks on a casein-based low-Fe diet containing less than $10 \mathrm{mg} / \mathrm{kg}$ Fe (Labsure Animal Foods, PO Box 6, Agrarian House, Castle Street, Poole, Dorset). No further control animals for this sub-group of Fe-deficient rabbits were used.

\section{Tissue criteria of Fe status}

Blood $(10 \mathrm{ml})$ ) was removed immediately before death and analysed for haemoglobin concentration and serum Fe by the methods of Drabkin \& Austin (1932) and Peters et al. (1956) respectively. Hepatic non-haem Fe was measured in tissue obtained post mortem by the method of Weinfeld (1964): it was related to hepatic protein as assayed by the fluorescamine method in the presence of $5 \mathrm{~g}$ sodium dodecyl sulphate/l (Van Frank, 1975).

\section{Measurement of whole body iron retention}

Whole body retention of ${ }^{59} \mathrm{Fe}$ was determined simultaneously in matched animals given $2 \mu \mathrm{mol}$ ferrous ascorbate or ferric nitrilotriacetate in $3 \mathrm{ml}$ neutral saline $(9 \mathrm{~g}$ sodium chloride/l) by gavage after an overnight fast. The value for $\mathrm{Fe}$ :chelate concentration was $1: 20$ and $1: 2$ for ferrous ascorbate and ferric nitrilotriacetate respectively. Radioactivity was measured in a twin-crystal small-animal body gamma counter and the emission was determined in four positions to correct for geometrical variation. The radioactivity from the animals was compared with a known standard at $1 \mathrm{~h}$ and periodically for $14 \mathrm{~d}$ after administration of the dose. The counting error was set to less than $1 \%$; the coefficient of variation due to geometrical factors was generally less than $5 \%$.

\section{Determination of intestinal Fe uptake}

Pairs of matched animals were killed after an overnight fast by injecting $30 \mathrm{ml}$ air rapidly into the circulation. The abdomen was opened immediately and the small intestine removed, flushed with ice-cold neutral saline and placed in incubation buffer. Appropriate segments of intestine were gently everted and full-thickness crescentic slices, weighing 5-15 mg, were cut from the duodenum ( $50 \mathrm{~mm}$ distal to the pylorus); jejunum $(600 \mathrm{~mm}$ distal to the pylorus); or ileum ( $200 \mathrm{~mm}$ proximal to ileo-caecal valve).

Uptake of ${ }^{59} \mathrm{Fe}$ (II) ascorbate or ${ }^{59} \mathrm{Fe}$ (III) nitrilotriacetate into duplicate slices was determined essentially as described previously (Cox \& Peters, 1979). Fe chelates were prepared fresh by adding tracer ${ }^{59} \mathrm{FeCl}_{3}$ (IFSI; Radiochemical Centre, Bucks.) to $50 \mu \mathrm{l}$ $1 \mathrm{M}$-sodium ascorbate or a similar volume of $0 \cdot 1$-M-sodium nitrilotriacetate at $\mathrm{pH} 3$. Maximum impurity of contaminating non-radioactive ferrous iron was $0.5 \mu \mathrm{mol} / 1$; it was not determined in the ferric chelates. Sufficient carrier- $\mathrm{Fe}$ as $\mathrm{FeCl}_{3}$ in $0.1 \mathrm{M}$-hydrochloric acid (5-15 $\mu \mathrm{mol})$ was added to give a specific radioactivity of $5-100 \mu \mathrm{Ci} / \mu \mathrm{g}$ atom $\mathrm{Fe}$. The chelate was diluted with $5 \mathrm{ml}$ warm buffer ( $20 \mathrm{~mm}$-Hepes, $130 \mathrm{~mm}-\mathrm{NaCl}, 3.5 \mathrm{~mm}$-potassium chloride, $10 \mathrm{~mm}$-D-glucose, $1 \mathrm{~mm}$-calcium chloride, $\mathrm{pH} 7 \cdot 35$ ) immediately before incubations were started. The chelate solutions were stable for at least $1 \mathrm{~h}$ : more than $98.5 \%$ passed through $0.22 \mu$ millipore filters; the radioactivity remained dialysable and, in the instance of the ferrous chelates, assay with o-phenanthroline reagent indicated no appreciable oxidation to the ferric form. ${ }^{57} \mathrm{Co}$ cyanocabalamin $\left(\mathrm{CT}_{12}\right.$; Radiochemical Centre) was also present as a non-absorbable marker of extra-cellular fluid at a final concentration of $10 \mu \mathrm{g} / \mathrm{ml}$ and specific radioactivity $30 \mu \mathrm{Ci} / \mu \mathrm{mol}$. The incubations, at $37^{\circ}$, were vigorously 
Table 1. Body-weights and iron status in experimental groups of rabbits

(Mean values with their standard errors)

\begin{tabular}{|c|c|c|c|c|c|c|c|c|c|}
\hline \multirow[b]{2}{*}{ Group $\dagger$} & \multirow[b]{2}{*}{$n$} & \multicolumn{2}{|c|}{ Wt (kg) } & \multicolumn{2}{|c|}{$\begin{array}{c}\text { Haemoglobin } \\
\text { concentration } \\
(\mathrm{g} / \mathrm{l})\end{array}$} & \multicolumn{2}{|c|}{$\begin{array}{c}\text { Serum Fe } \\
\mu \mathrm{mol} / 1\end{array}$} & \multicolumn{2}{|c|}{$\begin{array}{l}\text { Liver non-haem } \\
\mathrm{Fe} \text { (ng atom } / \mathrm{mg} \\
\text { protein) }\end{array}$} \\
\hline & & Mean & $\mathrm{SE}$ & Mean & $\mathrm{SE}$ & Mean & $\mathrm{SE}$ & Mean & $\mathbf{S E}$ \\
\hline Normal controls & 8 & $3 \cdot 22$ & 0.15 & 141 & 3 & 58.4 & 6.6 & 156 & 59 \\
\hline Chronic haemorrhage & 8 & $3 \cdot 13$ & $0 \cdot 13$ & $89^{* *}$ & 4 & $25 \cdot 9 * *$ & $4 \cdot 1$ & $43^{* *}$ & 7 \\
\hline Low-Fe diet & 3 & $1.82^{*}$ & $0 \cdot 14$ & $94^{*}$ & 7 & $10 \cdot 0^{*}$ & 3.7 & $11^{*}$ & 3 \\
\hline
\end{tabular}

Mean values were statistically significantly different from control values: ${ }^{*} P<0.05,{ }^{* *} P<0.01$.

$\dagger$ For details, see p. 252.

bubbled with pure oxygen and stopped as previously described: uptake was expressed as $\mathrm{pg}$ atoms $\mathrm{Fe} / \mathrm{unit}$ weight of intestine and in kinetic experiments as $\mathrm{pg}$ atoms/min per $\mathrm{mg}$ after $5 \mathrm{~min}$ incubation.

Statistical analysis

Differences between groups were assessed by $t$ tests for paired or unpaired observations. The significance of differences between groups where several stratified or matched sets of measurements were available was assessed by analysis of variance.

\section{RESULTS}

Fe status of experimental groups

The values are shown in Table 1. Controlled haemorrhage induced sideropenic anaemia which was maintained in spite of weight gains not significantly different from control animals. As shown, both haemorrhagic and nutritional sideropenic anaemia in the rabbits were associated with significantly reduced Fe stores in liver, when compared with control animals. As expected, the animals with Fe deficiency induced by diet gained little weight during the experimental period; the matched animals remained within $200 \mathrm{~g}$ of the mean weight during the period of controlled haemorrhage, and were not significantly different from matched controls.

\section{Whole body Fe retention}

Over-all absorption of the doses of ferrous and ferric chelates was judged in vivo by the measurement of Fe retention in control and bled rabbits (Fig. 1).

In normal control animals mean retention after $14 \mathrm{~d}$ of the dose of ferrous Fe was $1.5 \%$ of the dose and of the ferric dose $3.9 \%$ of the dose. Whole body retentions in the two matched pairs of bled animals were 28.2 and $17.8 \%$ of the dose of ferrous and ferric chelates. The results confirmed that absorption of chelated $\mathrm{Fe}$ in vivo was stimulated several fold in conditions of haemorrhagic Fe deficiency $(P<0.01)$.

Time course of intestinal Fe uptake and effects of metabolic poisons

As shown in Fig. 2, uptake of radio Fe by full-thickness slices continues incrementally over a prolonged incubation period in vitro, confirming previous observations with mucosal biopsy specimens of human duodenum (Cox \& Peters, 1979). Also displayed are the effects of metabolic poisons on $\mathrm{Fe}$ uptake: addition of fluoride and 2,4-dinitrophenol to this incubation mix progressively inhibited radio $\mathrm{Fe}$ influx. The uptake rate was reduced by $77 \%$ over the $30 \mathrm{~min}$ incubation period $(P<0.05)$. Comparable effects were observed on reducing the incubation temperature to $10^{\circ}$. 


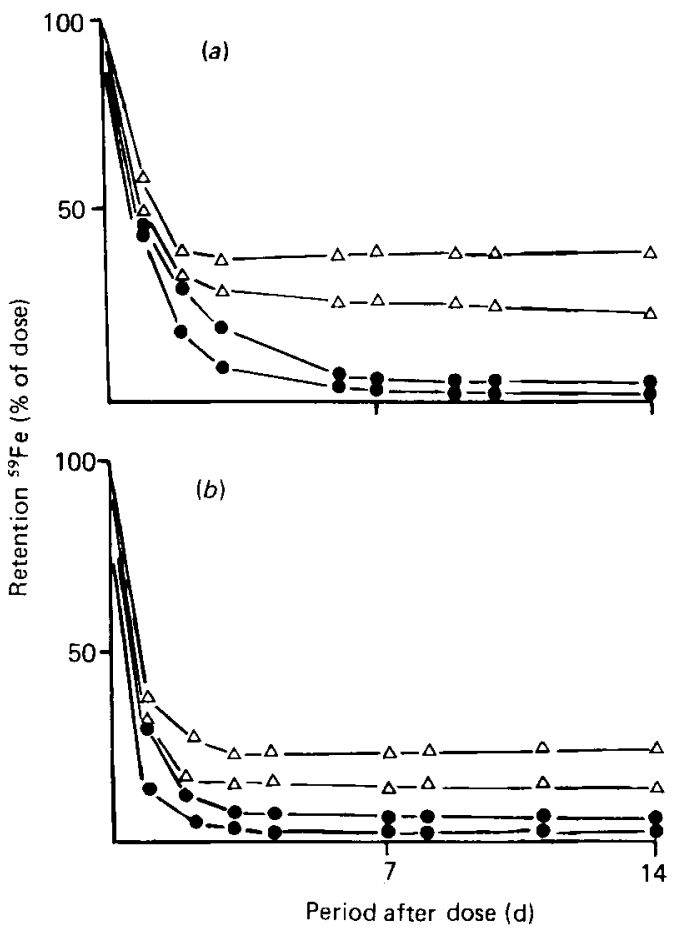

Fig. 1. Whole-body iron retention (\% of dose) in normal control $(\bullet)$ and Fe-deficient $(\triangle)$ rábbits given intragastric doses of $2 \mu \mathrm{mol}$ ferrous ascorbate $(a)$ or ferric nitrilotriacetate $(b)$. Each point represents a single determination on one animal; the difference between values for the control and chronically-bled animals was statistically significant $(P<0 \cdot 01)$.

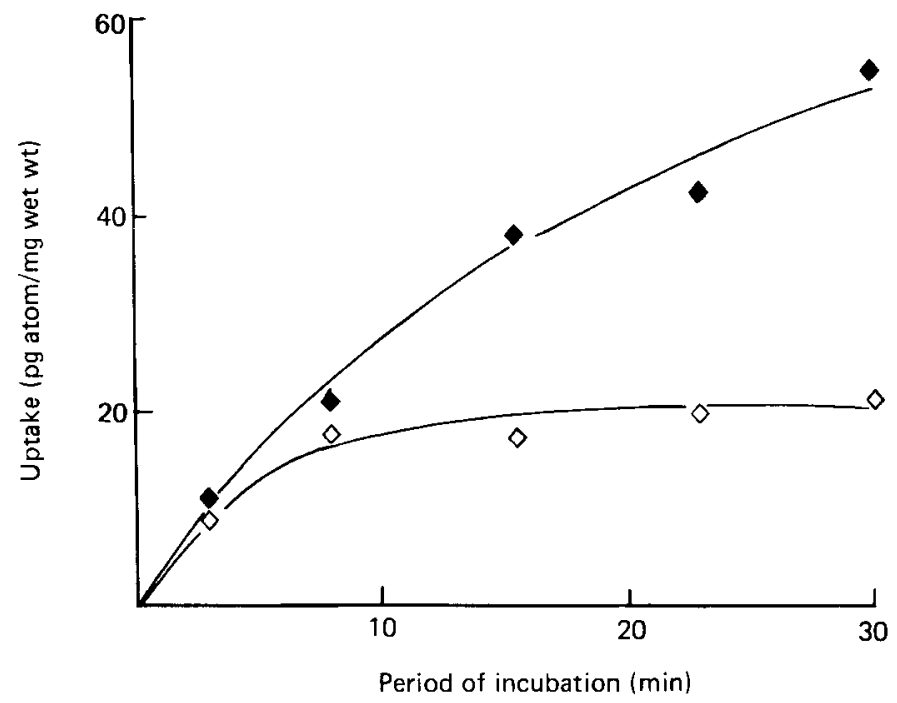

Fig. 2. Time-course of ${ }^{59} \mathrm{Fe}$ uptake (pg atom/ $\mathrm{mg}$ wet weight) by proximal intestinal slices from a normal control animal incubated in the presence of ferrous ascorbate at a concentration ot $25 \mu \mathrm{mol} / \mathrm{l}(\bullet)$. Uptake in the presence of $0.1 \mathrm{~mm}$-2,4-dinitrophenol and $10 \mathrm{~mm}$-sodium fluoride is shown $(\diamond)$. Each point represents the mean of duplicate determinations and the difference between values for the two treatments was statistically significant $(P<0.05)$. 


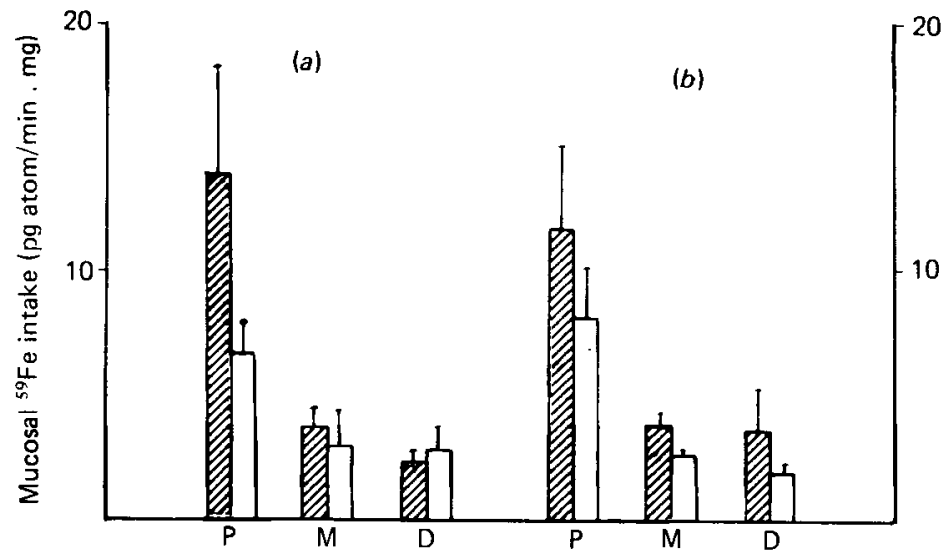

Fig. 3. Effect of location on mucosal ${ }^{59} \mathrm{Fe}$ uptake from $450 \mu \mathrm{mol} / 1$ ferrous ascorbate $(a)$ and $450 \mu \mathrm{mol} / 1$ ferric nitrilotriacetate $(b)$. Intestinal slices from proximal duodenum $(P)$, mid-jejunum $(M)$ and distal ileum (D) were incubated for $5 \mathrm{~min}$ (for details, see p. 252). (粼), Slices obtained from Fe-deficient animals; $(\square)$, obtained identically from normal control animals. Values are means with their standard errors for six paired determinations in duodenum and three paired determinations in distal intestine represented by vertical bars.

\section{Effects on location on intestinal Fe uptake}

Influx of $\mathrm{Fe}$ into intestinal slices obtained from distal intestine was substantially reduced when compared with slices from the duodenum (Fig. 3). This regional gradation was observed in the paired experiments employing both the ferrous and ferric chelates and was significant at $P<0.01$. In matched experiments employing intestinal slices from rabbits rendered chronically $\mathrm{Fe}$ deficient by controlled bleeding, influx of $\mathrm{Fe}$ was significantly enhanced but this effect was greatest in the proximal segments $(P<0.02)$. Influx of ferric $\mathrm{Fe}$ into slices of ileum was also stimulated in Fe deficiency; this effect was only evident in experiments employing ferric nitrilotriacetate.

\section{Kinetic studies of Fe uptake in conditions of Fe deficiency}

In order to examine further the nature of the modulations by Fe transport induced by conditions of body Fe deficiency, kinetic studies of uptake velocity over a range of medium concentrations of ferrous ascorbate were undertaken. Kinetic studies in normal control rabbits, chronically-bled animals, and a group rendered Fe-deficient by feeding a low-Fe diet are shown in Fig. 4. Duodenal uptake velocities in both groups of Fe-deficient animals were significantly enhanced when compared with duodenal slices from normal control animals. As previously shown in studies with mucosal biopsy specimens of human duodenum, initial $\mathrm{Fe}$ uptake velocities showed evidence of saturation within the range of concentrations used $(18-450 \mu \mathrm{mol} / \mathrm{l})$. Kinetic constants, calculated by linear regression of individual experiments from Eadie-Hofstee plots (Hofstee, 1957) are set out in Table 2. Both haemorrhagic and nutritional $\mathrm{Fe}$ deficiency induced comparable increases in the apparent maximum transport capacity for $\mathrm{Fe}$ influx with insignificant alterations in the apparent affinity constant $\left(K_{t}\right)$ for transport.

\section{DISCUSSION}

Fe absorption in mammals is regulated by the intestine and has long been known to increase adaptively in response to biological stresses including haemorrhage, nutritional Fe deficiency and pregnancy. However, the intestinal control mechanisms are incompletely understood 


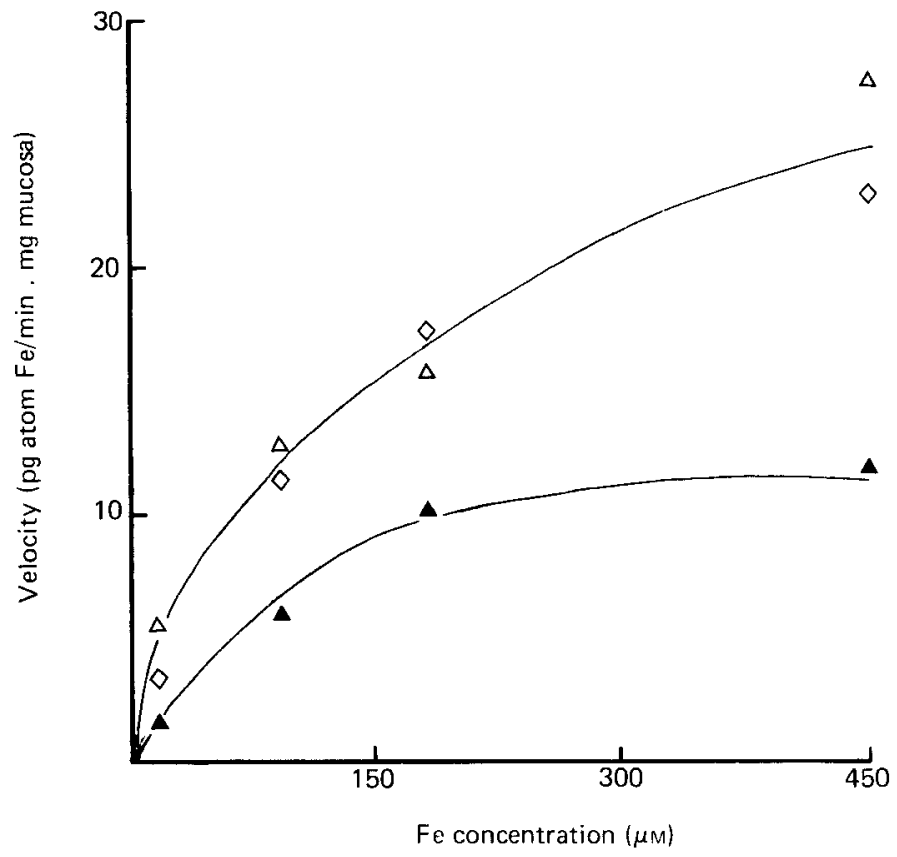

Fig. 4. Intestinal iron uptake kinetics. Uptake velocity (pg atom $\mathrm{Fe} / \mathrm{min}$ per $\mathrm{mg}$ mucosa) over a range of concentrations of ${ }^{50} \mathrm{Fe}$ ascorbate was determined in slices of proximal intestine from normal control animals ( $\Delta$ ) and pair-matched animals rendered Fe-deficient by controlled haemorrhage $(\triangle)$. Uptake kinetics in a group of nutritionally-Fe deficient rabbits are also shown $(\diamond)$. In normal and chronically-bled animals, each point represents the mean of duplicate determinations for five animals $(P<0.01)$; in the nutritionally-Fe-deficient group, each point represents the mean of duplicate determinations in three animals. Mean values were statistically significantly different from control values: for chronically-bled animals $P<0.01$; for nutritionally-Fe-deficient animals $P<0.05$.

Table 2. Kinetic constants of duodenal Fe uptake in iron-deficient rabbits

(Mean values with their standard errors)

\begin{tabular}{|c|c|c|c|c|c|}
\hline \multirow[b]{2}{*}{ Group $\dagger$} & \multirow[b]{2}{*}{$n$} & \multicolumn{2}{|c|}{$\begin{array}{c}V_{\max } \\
(\mathrm{pg} \text { atom } / \mathrm{min} \text { per } \mathrm{mg})\end{array}$} & \multicolumn{2}{|c|}{$\begin{array}{c}K_{\mathrm{t}} \\
(\mu \mathrm{mol} / \mathrm{l})\end{array}$} \\
\hline & & Mean & $\mathrm{SE}$ & Mean & $\mathbf{S E}$ \\
\hline Normal controls & 5 & $16 \cdot 1$ & $3 \cdot 4$ & 138 & 16 \\
\hline Chronic haemorrhagic anaemia & 5 & $27 \cdot 2^{*}$ & $4 \cdot 8$ & 98 & 17 \\
\hline Low-Fe diet nutritional anaemia & 3 & $25 \cdot 3$ & $7 \cdot 1$ & 110 & 8 \\
\hline
\end{tabular}

$V_{\max }$, maximum transport capacity; $K_{\mathrm{t}}$, apparent affinity constant for iron transport.

* Mean values were statistically significantly different from control values, $P<0 \cdot 025$.

$\uparrow$ For details, see experimental section (p. 252).

(Forth \& Rummel, 1973). Moreover, variations in Fe handling by different species of experimental animal as well as wide differences in methodology, especially in regard to measurement of absorption, have added confusion to the available literature (Cox, 1979).

In the present study attention has been focused on the regulatory significance of the initial step in the over-all absorption of $\mathrm{Fe}$, namely influx across the luminal surface of the mucosa. The use of a freely-diffusible non-absorbable marker and incubation periods short enough to avoid intra-cellular accumulation with reflux of radio $\mathrm{Fe}$ into the medium, allows valid 
measurements of initial uptake velocities of this probe to be made (Sallee et al. 1972; Cox $\&$ Peters, 1979). The use of divalent instead of trivalent Fe chelates additionally avoids binding of Fe to plasma transferrin entrapped within interstices at the cut surfaces of the intestinal slices: it is remotely possible that such binding could contribute to apparent uptake values in the presence of nitrilotriacetate chelates.

In the rabbit duodenum $\mathrm{Fe}$ uptake was inhibited powerfully by the addition of metabolic poisons to the incubation medium and, as in human duodenal mucosa, initial velocities showed evidence of saturation within a physiological range of Fe concentration. These properties are features shared by active transport processes where carrier-mediated fluxes are dependent on metabolically-derived electrochemical gradients. By analogy with regulation of biochemical pathways, such a transport process would be expected to be amenable to cellular control mechanisms, especially in view of its primary location in the absorptive sequence.

Over-all absorption and intestinal uptake of Fe presented as stable ferrous or ferric chelates were comparable, suggesting, in agreement with the experiments of Forth et al. (1965), that the biological availability of these redox states may be related to aqueous solubility and kinetic lability of individual complexes, rather than intrinsic atomic differences. Furthermore, the gradation of Fe uptake in the more distal segments of intestine was observed with both chelates. Absorption and uptake of both chelates were also stimulated significantly in conditions of haemorrhagic Fe deficiency.

In experimental $\mathrm{Fe}$ deficiency initial uptake velocities for $\mathrm{Fe}$ were markedly stimulated and the kinetic analyses indicated an enhanced $V_{\max }$ for transport with insignificant alterations in apparent affinity, or $K_{t}$, for Fe. These kinetic perturbations suggest that the intestinal $\mathrm{Fe}$ transport is stimulated by increased carrier activity rather than changes in carrier affinity.

Several carrier moieties for Fe have been localized to the mucosal epithelium, and the mucosal transferrin purified from epithelial cytosol in rodent intestine is an example of a transport protein whose synthesis is stimulated in conditions of Fe deficiency (see Hübers et al. 1976). Notwithstanding the existence of cytosolic Fe transport proteins, we have recently provided evidence that enhanced transfer of $\mathrm{Fe}$ across the intestinal brush border is mediated in the rabbit by transport moieties within the microvillus membrane. We have shown that $\mathrm{Fe}$ binding sites in membranes purified from $\mathrm{Fe}$-deficient animals were reversibly increased in number but that affinity for Fe was not altered when compared with membranes from normal control rabbits (Cox \& O'Donnell, 1980). The kinetic changes in membrane Fe binding were comparable to those observed in Fe transport into intestinal slices, and suggest that the two processes are linked functionally.

Many previous studies of intestinal $\mathrm{Fe}$ uptake neither examined authentic initial rates nor corrected for adherent, unabsorbed fluid. Acheson \& Schultz (1972) were the first to measure instantaneous rates of $\mathrm{Fe}$ influx into segments of rabbit duodenum: they demonstrated an enhanced influx in rabbits made Fe deficient by bleeding and used $1 \mathrm{mmol}$ ferrous ascorbate/ 1 . The increased $\mathrm{Fe}$ influx was restored to normal on repleting the animals with Fe parenterally. The present experiments confirm and extend these findings. Both nutritional and haemorrhagic anaemia induced comparable increases in influx of ferrous and ferric chelates and kinetic analysis revealed that this was related to stimulation of $V_{\max }$ for uptake, as had been previously observed in vitro in specimens of human duodenal mucosa (Cox \& Peters, 1980). In contrast, similar experiments employing everted gut segments and isolated epithelial cells from rat intestine failed to show any evidence for increased $\mathrm{Fe}$ influx rates in conditions of Fe deficiency, or any evidence of active transport (Sheehan, 1976; Savin \& Cook, 1978). Furthermore, Fe uptake by brush borders isolated from proximal rat intestine was not enhanced in preparations from Fe-deficient animals 
(Greenberger et al. 1969). The results strongly suggest that species differences account for these inconsistencies. Our observations in the rabbit support the view that Fe absorption may be controlled by regulation of the initial uptake of $\mathrm{Fe}$ by the intestine and is related to the activity of Fe-binding sites in the brush-border membrane. Fe deficiency would appear to be associated with an extension of the uptake capacity for $\mathrm{Fe}$ along the intestine and this, combined with enhanced transport per unit of intestine, would greatly increase over-all uptake and absorption.

Fe deficiency induced by haemorrhage or low-Fe diet represents a profound biological stress and rapidly stimulates compensatory responses in the intestine. In spite of this, the mechanism of $\mathrm{Fe}$ deficiency in the two circumstances is quite different: bleeding removes body $\mathrm{Fe}$ without affecting the availability of dietary $\mathrm{Fe}$ in the lumen; nutritional deficiency merely reduces the availability of lumen $\mathrm{Fe}$ below the concentrations necessary for absorption to match requirements. Irrespective of the stimulus, the changes in intestinal Fe uptake are the same: the capacity of the uptake process is increased without material changes in affinity. It is remotely possible that, owing to differences in formulation, other dietary factors in the nutritionally $\mathrm{Fe}$-deficient group may have modified $\mathrm{Fe}$ uptake. Although these effects cannot be ruled out, the similarities of uptake response in the two groups of anaemic animals make such a possibility unlikely.

As has been pointed out in the instance of human nutrition (Cox, 1980) enhanced carrier capacity under conditions of reduced bioavailability may only result in modest enhancement of over-all $\mathrm{Fe}$ absorption since at low luminal concentrations full exploitation of the increased capacity of the intestine could not occur. The behaviour of the rabbit intestine in vitro appears to match that of human duodenal mucosa closely, and, if parallel behaviour can be assumed in vivo, these considerations would partially account for the prevalence of Fe deficiency in human populations occurring in spite of intestinal compensatory mechanisms.

This work was supported by the Wellcome Trust. The authors wish to thank Alex Ranicar and Terence Spinks for help with the whole body counter, and Mrs Berny Owens and Miss Ann Love for typing the manuscript.

\section{REFERENCES}

Acheson, L. S. \& Shultz, S. G. (1972). Biochim. biophys. Acta. $255,479$.

Cox, T. M. (1979). The regulation of iron absorption by the digestive tract. MD Thesis, University of London. Cox, T. M. (1980). N. Engl. J. Med. 302, 695.

Cox, T. M. \& O'Donnell, M. W. (1980). Biochem. Int. 1, 466.

Cox, T. M. \& Peters, T. J. (1979). J. Physiol., Lond. 289, 469.

Cox, T. M. \& Peters T. J. (1980). Br. J. Haemat. 44, 75.

Drabkin, D. L. \& Austin, J. H. (1932). J. Biol. Chem. 98, 719.

Forth, W., Lichtenberg, H. \& Rummel, W. (1965). Arch. Exp. Path. Pharmacol. 250, 234.

Forth, W. \& Rummel, W. (1973). Physiol. Rev. 53, 724.

Greenberger, N. J., Balcerzak, S. P. \& Ackerman, G. A. (1969). J. Lab. Clin. Med. 73, 711.

Hofstee, B. H. J. (1957). Science, N.Y. 116, 329.

Hübers, H., Hübers, E., Rummel, W. \& Crichton, R. (1976). Eur. J. Biochem. 66, 447.

McCance, R. A. \& Widdowson, E. M. (1937). Lancet 233, 680.

Peters, T., Giovaniello, T. J., Apt, L., Ross, J. F. \& Trakas, A. P. (1956). J. Lab. Clin. Med. 48, 280.

Sallee, V. L., Wilson, F. A. \& Dietschy, J. M. (1972). J. Lipid Res. 13, 184.

Savin, M. A. \& Cook, J. D. (1978). Gastroenterology 75, 688.

Sheehan, R. G. (1976). Am. J. Physiol. 231, 1438.

Van Frank, R. M. (1975). Analyt. Biochem. 65, 552.

Weinfeld, A. (1964). Acta med. Scand. 247, suppl. 13. 